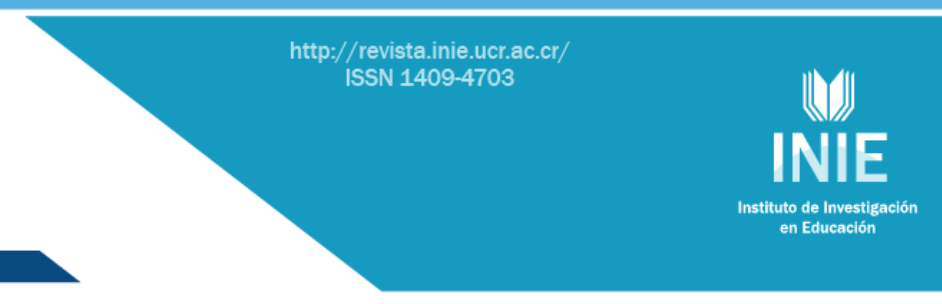

\title{
LA ORIENTACIÓN DEL APRENDIZAJE PARA LA TOMA ESTRATÉGICA DE APUNTES EN ESTUDIANTES UNIVERSITARIOS LEARNING ORIENTATION FOR TAKING STRATEGIC NOTES ON UNIVERSITY STUDENT
}

\section{Volumen 15, Número 1}

Enero - Abril

pp. $1-28$

Este número se publicó el $1^{\circ}$ de enero de 2015

DOI: dx.doi.org/10.15517/aie.v15i1.16975

Isaac Iran Cabrera Ruiz

Yoisel Eguino de la Paz

Mirelis Jiménez González

Revista indizada en REDALYC, $\underline{\text { SCIELO }}$

Revista distribuida en las bases de datos:

CATÁLOGO DE LATINDEX, IRESIE, CLASE, DIALNET, DOAJ, E-REVIST@S, SHERPA/ROMEO, QUALIS, MIAR

Revista registrada en los directorios:

ULRICH'S, REDIE, RINACE, OEI, MAESTROTECA, PREAL, CLACSO 


\title{
LA ORIENTACIÓN DEL APRENDIZAJE PARA LA TOMA ESTRATÉGICA DE APUNTES EN ESTUDIANTES UNIVERSITARIOS LEARNING ORIENTATION FOR TAKING STRATEGIC NOTES ON UNIVERSITY STUDENT
}

\author{
Isaac Iran Cabrera Ruiz' \\ Yoisel Eguino de la Paz \\ Mirelis Jiménez González ${ }^{3}$
}

\begin{abstract}
Resumen: El artículo científico fundamenta teórica y metodológicamente un sistema de orientación para la toma estratégica de apuntes, a partir de la teoría planificada de las acciones mentales que integra la concepción de aprendizaje estratégico, desde un enfoque del sujeto que aprende, así como la concepción didáctica del proceso de aprendizaje. El sistema para la toma estratégica de apuntes se estructura en 3 bases orientadoras de la acción, que contienen una síntesis representacional de la acción y las condiciones de su realización con respecto al enfoque estratégico en el aprendizaje, el uso de técnicas y la perspectiva estratégica para tomar apuntes. Contiene, además, indicaciones metodológicas para la orientación de la toma estratégica de apuntes que abarca la lógica, lo pedagógico y lo psicológico. Los resultados forman parte de una investigación realizada en la Universidad Central "Marta Abreu" de Las Villas en Santa Clara, Cuba, en 2013. El sistema de orientación fue evaluado por criterio de evaluadores externos, incluyéndose las mejoras correspondientes. Constituye, como resultado científico, la plataforma para elaborar medios de enseñanza dirigidos al desarrollo de la toma estratégica de apuntes.
\end{abstract}

Palabras clave: ORIENTACIÓN DEL APRENDIZAJE, ESTRATEGIAS DE APRENDIZAJE, TOMA DE APUNTES, DOCENCIA UNIVERSITARIA,CUBA

\begin{abstract}
The scientific paper theoretically and methodologically based system for strategic guidance note taking from the theory of planned actions that integrates mental conception of strategic learning from a perspective of the learner and the learning design process learning. The system for strategic note-taking is organized in 3 guiding action bases containing a representational synthesis of action and the conditions of its performance against the Strategic approach to learning, the use of technical and strategic perspective to take notes. It also contains methodological guidance for targeting strategic note taking covering logic, pedagogical and psychological. The results are part of a research conducted at the Central "Marta Abreu" of Las Villas University in Santa Clara, Cuba in 2013. Guidance system was evaluated by external evaluators introduced criterion corresponding improvements. It is, as a scientific result, the platform for the development of teaching aids aimed at developing strategic note taking.
\end{abstract}

Keywords: LEARNING ORIENTATION, LEARNING STRATEGIES, TAKING NOTES, UNIVERSITY TEACHING, CUBA

\footnotetext{
${ }^{1}$ Doctor en Ciencias Pedagógicas, Profesor de la Facultad de Psicología de la Universidad Central "Marta Abreu" de Las Villas. Dirección electrónica isaacicr@uclv.edu.cu

2 Profesor de la Facultad de Psicología de la Universidad Central "Marta Abreu" de Las Villas, Cuba. Licenciado en Psicología.

3 Subdirectora académica y profesora del Centro de Superación para la Cultura, Villa Clara, Cuba. Licenciada en Filología.
}

Artículo recibido: 23 de junio, 2014

Enviado a corrección: 18 de julio, 2014

Aprobado: 20 de octubre, 2014 


\section{Introducción}

La toma de notas o apuntes constituye el recurso por excelencia que utilizan los estudiantes universitarios en sus procesos de aprendizaje. Múltiples investigaciones muestran la importancia del enfoque estratégico en esta actividad. Autores como Peper y Mayer (1978, 1986, citados en Monereo y Pérez, 1996), señalan cómo estas pueden incrementar la atención y el interés estudiantil sobre el tema tratado, estimulan el nivel de procesamiento de la información a un nivel más acabado, y facilitan la vinculación del contenido con conocimientos previos del estudiante. Asimismo, Espino y Miras (2010) demostraron la correlación existente entre el nivel de elaboración personal de los apuntes y los resultados en el aprendizaje, donde a mayor nivel de elaboración, mejores resultan las calificaciones alcanzadas.

Monereo y Castelló (1999) consideran que los estudiantes estratégicos suelen ajustar su conducta a un conjunto de condiciones tales como el tipo de evaluación que se implementa en la asignatura, a la existencia o no de base material de estudio, la naturaleza del contenido dado, la importancia relativa de la materia y el interés o novedad de la temática tratada. Estos estudiantes recuperan sin dificultad la información correspondiente a determinada unidad temática ante la demanda de su recuerdo, y suelen estudiar sus apuntes días antes de realizar una prueba, lo cual les sirve para recomponer los contenidos recibidos en clase, convirtiéndose sus apuntes en un extracto que establece rápidamente conexión con los datos almacenados en la memoria.

A pesar de la significación analizada de la toma de apuntes en el aprendizaje, predomina en los estudiantes universitarios la carencia de un enfoque estratégico con respecto a la toma de apuntes o notas, según demuestran Monereo y Pérez (1996).

En esta dirección, el estudio de caso realizado por Eguino (2013), ${ }^{4}$ con una muestra constituida por 9 estudiantes universitarios de la Facultad de Psicología de la Universidad Central "Marta Abreu" de las Villas, específicamente de la carrera de Psicología y pertenecientes al 2do, 3er y 4to año de la carrera, arrojó como principales regularidades:

- Los estudiantes tienden a anotar de forma exhaustiva todo lo que el profesorado da en clases y no una síntesis del discurso de este último, lo que lleva a que la copia rápida del material expuesto en clases se convierta una herramienta clave para guardar la mayor información posible.

\footnotetext{
${ }^{4}$ El estudio realizado por Eguino bajo la dirección de Cabrera, constituye el referente para la elaboración del artículo. En él se obtuvieron tanto los resultados del estudio de caso, como el sistema de orientación para la toma estratégica de apuntes que se presenta más adelante.
} 
- Entre las técnicas para tomar apuntes, constituyen las más utilizadas la elaboración de resúmenes y la anotación o el resaltado de palabras claves, por el ahorro en tiempo y espacio.

- Se identificó que la organización de la información, la ampliación de los apuntes, la reflexión sobre lo anotado y el garantizar la calidad de las notas de forma general, no son muy utilizadas por los estudiantes. Dentro de ellas, la organización de la información y la reflexión sobre lo anotado son las más empleadas; la primera destaca temáticas generales y hace uso, además, de la señalización de la información más importante mediante el subrayado; la segunda, a través de la revisión de lo anotado y la corrección de posibles incoherencias, además del planteamiento de interrogantes al respecto.

Estos resultados evidencian que el alumnado estudiado no seguía una actuación estratégica al tomar apuntes, lo cual condujo a la proyección en la necesidad de contribuir al desarrollo de estrategias de aprendizajes en estudiantes universitarios.

Precisamente, las estrategias de aprendizaje han devenido en un campo de estudio importante de las ciencias como la Psicología y la Pedagogía, en sus respectivas contribuciones a la formación de profesionales. El conocimiento científico y tecnológico progresa geométricamente y su introducción en los procesos productivos de las grandes empresas es rápida, acortándose la vida útil de tecnologías y productos. Incluso, como señala Tünnermann (2010), para la mayoría de los empleos de alta calificación, lo aprendido en la etapa de formación tiene hoy una vida útil de entre 5 y 10 años.

En este sentido, como refiere Cabrera (2009), la acelerada obsolescencia del conocimiento y la imposibilidad de ampliación infinita y marcha sincrónica del currículo ${ }^{5}$ al ritmo que la ciencia reclama, en la formación profesional, el desarrollo de conocimientos pertinentes $^{6}$ con el empleo, según Menchinskaya y Sabutorova (1988), de métodos de enseñanza que permitan el más alto desarrollo intelectual y la adquisición de habilidades para alcanzar conocimientos de forma independiente.

De hecho, el alumnado universitarios no solo se apropian de la cultura a partir de procesos de aprendizaje que les permiten el dominio progresivo de los objetos y sus usos, de los modos de actuar, de pensar y de sentir, sino también, como señalan Castellanos, D.,

\footnotetext{
${ }^{5}$ Los conocimientos científicos y tecnológicos no pueden ser incorporados a los planes de estudio al mismo ritmo en que se producen.

${ }^{6}$ Morin (1999) los identifica como conocimientos contextualizados, globales, multidimensionales y complejos.
} 
Castellanos, B., Llivina y Silverio (2001), de las formas de aprender vigentes en cada contexto histórico.

El alumnado necesita aprender a aprender durante el proceso formativo, apropiarse, junto de los conocimientos pertinentes que tributen al modelo del profesional, de conocimientos que permitan el aprendizaje de esos conocimientos pertinentes y de los nuevos a los que tendrá que acceder durante su vida. Siguiendo a Santana (2009), es posible percatarse de que la idea del aprendizaje autónomo y las estrategias de aprendizaje no es tan reciente como suele pensarse, pues el principio de aprender a aprender, ya constituía en la década de 1970, un propósito para el trabajo en los distintos niveles educativos ante el fracaso escolar.

Para denominar el reajuste que requiere el aprendizaje a tono con las actuales demandas, González, M. A. (2012) identifica una serie de expresiones que han sido acuñadas: autoaprendizaje, autoeducación, autodirección, metacognición, aprendizaje autogestionado, aprendizaje autorregulado, aprendizaje andragógico, aprendizaje planificado, aprendizaje autoorganizado y aprendizaje autónomo. La idea central que puede extraerse de estas preocupaciones es que el proceso de enseñanza aprendizaje debe tributar al desarrollo de la autonomía, el crecimiento personal y el profesional.

De este modo, el tema de las estrategias de aprendizaje ha emergido en el contexto científico como una necesidad transversal al proceso de enseñanza aprendizaje, ganando en relevancia y significación, lo cual es sistematizado por Marugán, Martín, Catalina y Román (2013), al sistematizar las producciones de autores en torno a las categorías de análisis, aportaciones, diseño de programas y pautas de entrenamiento y trabajos de investigación, alrededor de las cuales agrupa a los autores más representativos.

Las definiciones con respecto a las estrategias de aprendizaje evidencian el énfasis en la esfera cognitiva del sujeto, incluso cuando se contempla la motivación. Entre estas aportaciones, como señalan Nieves, Otero y Molerio (2007), se ha impuesto la concepción constructivista en el ámbito universitario, como alternativa en los planteamientos educativos para el desarrollo de la autonomía en el aprendizaje, desde un enfoque estratégico de la enseñanza y el aprendizaje.

Es cierto, según apuntan las autoras, el predominio de la orientación hacia el cognitivismo, superado en la comunidad discursiva de nuestros contextos educativos, pero arraigado en las prácticas educativas. De este modo, se revela, no la necesidad de renunciar al enfoque estratégico, sino a un adecuado posicionamiento en el sujeto que aprende, en la 
unidad de la cognitivo y lo afectivo, donde la autonomía, como señalan Nieves, Otero y Molerio (2007), supone madurez en la intencionalidad del sujeto en su formación personal y profesional, que trascienda la autoeducación. Sin embargo, ello no amerita desconocer el papel de la infraestructura cognitiva como configuración de la personalidad en la autoeducación, ni su papel en el enfoque estratégico como elemento de autonomía para aprender.

\section{Fundamentos teórico metodológicos para la orientación de la toma estratégica de apuntes en estudiantes universitarios}

\subsection{Aprendizaje estratégico}

La tesis fundamental en el enfoque constructivista con respecto a las estrategias de aprendizaje, consiste en el énfasis utilitario para la optimización del funcionamiento de los procesos cognitivos, o como lo escribió Poggioli (1989), influir en cómo los individuos seleccionan, adquieren, retienen, organizan e integran nuevos conocimientos.

Desde los planteamientos de autores como Coll (1996), Palma (1997), Pozo y Monereo (1999), el aprendizaje estratégico es un proceso de activación de los procesos cognitivos en función de la adquisición de conocimientos de manera significativa, donde el sujeto actúa de modo metacognitivo como momento de regulación de su desempeño intelectual. Ser estratégico en el aprendizaje implica la reflexión sobre el aprendizaje para perfeccionar el proceso cognitivo seguido, así como la intencionalidad y decisión con respecto a las exigencias y demandas en el proceso de aprendizaje.

Como ya señalamos, este enfoque amerita un posicionamiento del aprendizaje estratégico en el sujeto que aprende, así como una diferenciación entre aprendizaje estratégico y autonomía en el aprendizaje.

La autonomía no puede reducirse al aprendizaje estratégico, aunque este sería una dimensión de aquella. Tener autonomía en el aprendizaje implica, entre otros aspectos, como plantean Nieves, Otero y Molerio (2007), el desarrollo de la autoeducación como proceso intencional del sujeto, dirigido al autoperfeccionamiento, a la estructuración y reestructuración de la realidad personal y de la representación que la persona tiene sobre sí misma; presupone ciertos niveles de madurez y desarrollo de la personalidad, revelándose la edad juvenil como su periodo sensitivo, donde el sujeto deviene en autogestor de su propio desarrollo. 
Ahora bien, el enfoque estratégico en el aprendizaje implica comprender que lo cognitivo forma parte de complejos sistemas de operaciones inconscientes, que en sus formas superiores se organizan en lo que González, F. (1997) denomina infraestructura cognitiva del sujeto, considerándola como organización de elementos estructurales relacionados con las formas superiores de actividad cognitiva que intervienen en la producción y organización de la información, a partir de la expresión intencional del sujeto, la cual tiene en su base los estilos cognitivos, las capacidades y las diversas formas de organización y producción de la información a nivel cognitivo.

De este modo, las formas superiores de actividad cognoscitiva pertenecen a un sujeto cognoscente, expresión de la personalidad como sujeto de su actividad, conteniendo la unidad de los cognitivo y lo afectivo, ya que "el proceso cognoscente de la realidad no se puede separar del momento vivencial, emocional, de la relación del hombre con el medio, a través del cual se produce este conocimiento" (González, F. 1986, p. 35).

La comprensión del aprendizaje estratégico como dimensión del sujeto, posibilita superar los reduccionismos en la absolutización de las estrategias de aprendizaje como elemento cognitivo, redimensionándolas como formas superiores de actividad cognoscitiva del sujeto.

Desde esta perspectiva personológica, resultan claves los aspectos teóricos aportados por Monereo (1990), Monereo, Castelló, Clariana, Palma y Pérez (1997), con respecto a las estrategias de aprendizaje y que se sistematizan a continuación:

- Son procesos de toma de decisiones (conscientes e intencionales)

- Se eligen y recuperan conocimientos para cumplimentar una determinada demanda u objetivo de aprendizaje

- Se aplican métodos o procedimientos

- Se consideran las características de la situación educativa en que se produce la acción

El aprendizaje estratégico alude a la adquisición de conocimientos a través del empleo de estrategias en el proceso de aprendizaje. Las estrategias de aprendizaje se estructuran sobre la base de un conocimiento condicional o estratégico, y se expresan a través de la actuación estratégica.

El conocimiento implica un plano reflexivo valorativo expresado a través de la reflexión del sujeto; es una categoría que contiene el producto de la relación del sujeto con la realidad como elaboraciones subjetivas, y en sus formas más complejas actúa, según González, F. 
(1986), como un sistema de relaciones entre conceptos ya formados, representando así un elemento estable que el sujeto activamente opera y utiliza. Así, el conocimiento condicional o estratégico contiene información sobre las situaciones (cuándo, por qué) en que determinado conocimiento debe activarse para conseguir el objetivo propuesto, guiando el proceso de toma de decisiones entre diversos cursos de acción, en correspondencia a determinado objetivo y en un contexto concreto.

La actuación estratégica hace referencia a la expresión del conocimiento condicional, a la puesta en práctica de estrategias de aprendizaje a través de un conjunto de acciones necesarias y suficientes que realiza el sujeto durante su comportamiento.

Así, el aprendizaje estratégico está determinado por las mediaciones del conocimiento condicional o estratégico sobre los conocimientos conceptuales o declarativos (hechos, conceptos, principios), los procedimentales (componentes prácticos del saber, las acciones) y los actitudinales (formas de relación con el mundo) en el proceso de aprendizaje, al utilizar el sujeto intencionalmente el conocimiento de forma estratégica.

El aprendizaje estratégico es una forma de aprender mediante la toma consciente e intencional de decisiones sobre los procedimientos más adecuados en la resolución de una tarea, a partir de la reflexión del sujeto que aprende, lo cual implica un conocimiento sobre determinados procedimientos y su empleo, así como su uso ajustado a las exigencias contextuales y de contenido, y a los continuos cambios que se producen en la actividad.

Otra de las características significativas del aprendizaje estratégico es la regulación del sujeto sobre el proceso de aprendizaje, que se configura como componente metacognitivo en la estrategia de aprendizaje, el cual se caracteriza, siguiendo a Monereo, et al. (1997) como un conjunto de autointerrogantes que el alumnado se hace y responde:

1. Planificación, antes de iniciar la tarea: ¿Cuáles son las condiciones relevantes de la tarea que he de aprender o debo resolver? ¿Qué resultado o producto debo obtener?¿Cómo se evaluará? ¿Qué conocimientos previos pueden ayudarme a enfrentar la tarea? ¿Qué técnicas o procedimientos pueden ayudarme a lograr los objetivos?

2. Autorregulación (supervisión mientras se realiza la tarea): ¿Me estoy ajustando a las condiciones de la tarea? ¿Estoy acercándome al resultado esperado, en los términos apropiados? ¿Estoy superando los obstáculos que van apareciendo? ¿Doy respuesta a las dudas y dificultades con las que tropiezo? Las técnicas o procedimientos que estoy 
aplicando, ¿me permiten avanzar en la dirección deseada?, ¿qué otros procedimientos podría emplear?, ¿por qué no los utilizo?

3. Autoevaluación (una vez finalizada la tarea): ¿He respetado las condiciones que exigía la tarea?; si volviese a realizar una tarea parecida, ¿en relación con qué condiciones y de qué modo ajustaría mejor mi conducta? ¿He alcanzado los resultados esperados?; si volviese a efectuar esta tarea, ¿cómo interpretaría la demanda y los criterios de evaluación? ¿He resuelto satisfactoriamente las dificultades encontradas?; en otra ocasión, ¿cómo actuaría? ¿He utilizado las técnicas o procedimientos más apropiados?; en una nueva oportunidad, ¿qué procedimientos escogería?

\subsection{Orientación del aprendizaje}

El aprendizaje permite al individuo apropiarse de los contenidos y las formas de pensar, sentir y actuar construidas en la experiencia sociohistórica, con el fin de adaptarse a la realidad y transformarla. Aprender incluye siempre una actividad de asimilación que tiene lugar en condiciones concretas organizadas como parte de un proceso intencionado de enseñanza: el aprendizaje

La teoría de la formación por etapas de las acciones mentales considera el estudio como un sistema de determinados tipos de actividad, cuyo cumplimiento conduce al alumnado a los nuevos conocimientos y hábitos, donde el aprendizaje tiene lugar a través de acciones que posibilitan la asimilación del objeto de conocimiento. La actividad práctica del ser humano es la base del conocimiento, por lo tanto, existe una relación dialéctica entre acciones mentales y actividad práctica, de modo que las acciones mentales emergen de la actividad práctica, la cual mediatizan. Las acciones mentales son acciones subjetivas, la reproducción en el plano de las imágenes de los rasgos esenciales de acciones materiales.

La acción, siguiendo a Talízina (1988), se divide de acuerdo con su función, en 3 partes: orientadora, ejecutora y control. La parte orientadora de la acción se relaciona con la utilización por el sujeto, del conjunto de condiciones concretas, necesarias, para el exitoso cumplimiento de la acción dada. La parte ejecutora asegura las transformaciones dadas al objeto (ideal o material). La parte de control está dirigida a seguir la marcha de la acción, a confrontar los resultados obtenidos con los modelos dados, interviniendo en la corrección necesaria tanto en la parte orientadora como en la ejecutora de la acción.

Precisamente, la parte orientadora "constituye la instancia directiva y, en lo fundamental, de ella depende la calidad de la ejecución" (Galperin, 2000, p.183). Idea 
confirmada en las investigaciones desarrolladas por Réstaetova, Galperin y Pántina, Zaporózhets (citados en Talízina, 1988), donde se demostró que el papel decisivo en la formación de la acción es la parte orientadora, que está dirigida a la construcción correcta y racional de la parte ejecutora, y a asegurar la elección racional de uno de los posibles cumplimientos.

La idea central de esta teoría se resume en que lo propiamente psicológico de la actividad es la orientación, la cual expresa anticipación de la ejecución y su dirección, donde la base de los procesos psíquicos y sus cualidades es la acción mental. En este sentido, es posible sistematizar los principales planteamientos de Galperin (1982):

- El sujeto tiene la capacidad de orientarse por sí mismo y de aprender a orientarse por los indicadores de instrumentos.

- La actividad orientadora se hace indispensable en los casos en que los mecanismos que existen o la propia la acción estereotipada son insuficientes y se necesita señalar de nuevo la acción, o adaptarla a las condiciones de existencia. Es decir, el sujeto requiere la actividad orientadora cuando en una situación dada no existen las condiciones que automáticamente garantizan el éxito.

- La regulación del comportamiento en determinadas situaciones es posible sobre la base de la imagen de las situaciones.

- La actividad orientadora garantiza la adaptación exitosa de las acciones a las situaciones individualmente variables, convirtiéndose en un eslabón clave en el proceso de enseñanza, en la formación de nuevas acciones.

- Las acciones que el sujeto dirige sobre la base de la orientación en el plano de la imagen son actos de conducta.

La actividad orientadora consiste en realizar, por parte del sujeto, "un examen de la nueva situación, confirma o no el significado racional o funcional de los objetos prueba y modifica la acción, traza un nuevo camino" (Galperin, 1982, p. 54), para más adelante llevar a cabo un control efectivo de su realización, en correspondencia con los indicadores establecidos.

La concepción de Galperin $(1982,1983)$ es posible sintetizarla en la idea de que el sujeto tiene la capacidad de dirigir sus acciones sobre la base de una imagen del campo de estas acciones, las cuales están dirigidas a un objetivo y se regulan a partir de la imagen de 
la situación. Es decir, el sujeto realiza acciones dirigidas a fines, por la representación que posee de la situación.

Ahora bien, la formación de la acción mental requiere un sistema de condiciones que posibilite la asimilación de las cualidades indispensables de la acción. Condiciones que Martínez (2003) sistematiza como formación de una adecuada motivación de las acciones, aseguramiento de la correcta ejecución de la nueva acción, formación de las propiedades deseadas de la nueva acción, y la traslación de la acción al plano mental.

El cumplimiento de la acción por el sujeto presupone la representación de esta acción, así como de las condiciones en que se cumple. Relación que origina la base orientadora de la acción como elemento estructural sobre el que transcurre la dirección de la acción concreta. Precisamente, en la formación de la acción, la parte orientadora determina la rapidez de la formación y la calidad de la acción que se logra con la formación de la base orientadora de la acción.

La base orientadora de la acción representa, según Talízina (1988), el sistema de condiciones en que realmente se apoya el sujeto al cumplir la acción, implica como puntualizó Galperin, una imagen de la acción por realizar y de las condiciones en que se realizará, es "el conjunto de condiciones según las que, de hecho, se orienta el alumnado durante el cumplimiento de la acción" (2003, p. 251).

La parte orientadora de la acción, según Martínez (2003), tiene dos sistemas: el motivacional y el operacional.

El sistema motivacional está destinado a dar un sentido personal a la ejecución de la acción, hacer al sujeto vivenciar la necesidad, la significación de la realización de determinada acción. En esta aun no entra ningún tipo de acción, y se plantea la necesidad de brindar una motivación positiva a la actividad, de forma contraria, quedarían comprometidas el resto de las etapas.

El sistema operacional constituye la representación de la composición de la acción antes de realizarla, y comprende el conocimiento del objetivo de la acción y la forma en que se obtendrá el resultado y los elementos de apoyo necesarios.

De este modo, mediante la representación anticipada de la tarea, se conforma el plan de la futura acción y así las bases para su dirección. Esta base orientadora tiene la propiedad de que cuando se da de forma que otorga las normas generales en función de la realización de determinado ejercicio, el sujeto ya será por sí mismo, capaz de formar sus 
propias bases orientadoras ante tareas similares y, por lo tanto, orientarse de forma individual ante la acción.

La actividad orientadora tiene como elementos fundamentales las imágenes y las acciones. Imagen de la acción y la del medio donde se realiza la acción, se unen en un elemento estructural único, sobre cuya base transcurre la dirección de la acción. La base orientadora de la acción posibilita la construcción correcta y racional de la parte ejecutora, y asegura la elección racional de uno de los posibles cumplimientos.

El contenido de la base orientadora sirve a los sujetos en el cumplimiento de la parte orientadora de la acción. No es la forma en que está representada la base orientadora de la acción lo que determina su eficacia, sino "el grado de generalización de los conocimientos que forman parte de ella (puntos de orientación) y de la plenitud del reflejo en ellos de las condiciones que determinan objetivamente el éxito de la acción y de cómo es recibida" (Talízina, 1988, p. 88).

\subsection{La base orientadora de la acción como dispositivo metodológico para el aprendizaje estratégico de la toma de apuntes}

La base orientadora de la acción implica una imagen de la acción por realizar y de las condiciones en las cuales va a realizarse la acción. Su elaboración como dispositivo metodológico para el aprendizaje, debe contener las unidades fundamentales del material del área dada y las reglas generales de sus combinaciones en fenómenos concretos, mostrando el cuadro de las circunstancias, el esbozo del plan de acción, el control y la concreción de su ejecución.

La base orientadora de la acción debe dar cuenta de las acciones más específicas que le confieren un carácter preciso, continuo, lógico y consecutivo a la acción que orienta. En su elaboración debe especificarse un conocimiento declarativo (imagen de la acción por realizar) donde se transmita la imagen de la acción, ideas, nociones de lo que debe hacer, así como por un conocimiento procedimental (condiciones en las cuales va a realizarse la acción) que muestre las acciones que en un continuo posibiliten la ejecución, el cumplimiento de la acción general.

Los componentes de la base orientadora deben ser unidades fundamentales del material del área dada, y contener las reglas generales de sus combinaciones en fenómenos concretos. El esquema de la base orientadora de la acción es un esquema operativo y 
resume de forma estructurada y comprensible para el sujeto, el conjunto de los siguientes aspectos, como sintetiza Domínguez (2005):

- La representación del producto final de la acción y sus índices cualitativos

- La representación de los productos parciales y sus índices cualitativos, así como el orden de ejecución de las acciones

- Las operaciones que permiten llegar a cada producto particular y el orden de realización de estas o algoritmo

- Las características de los tipos de materiales

- La selección de los instrumentos, ya sean materiales o ideales

- Las características de los medios y modos de control de la acción.

La generalización del contenido orientador de la base está determinada por el empleo de las invariantes del conocimiento para la ejecución de la acción, como se muestra en la Figura 1. Ahora bien, su asimilación dependerá de la relación entre las posibilidades objetivas de aplicación del conocimiento y las posibilidades subjetivas del individuo en cuanto a dicha aplicación. Es el contenido de la base orientadora de la acción, lo que determina el proceso de generalización a partir de los puntos de orientación que brinda al sujeto. Lo importante en la asimilación de los puntos de orientación con un carácter generalizado, es que el sujeto no aprenda de memoria los conocimientos que contiene, sino que logre utilizarlos para ejecutar la acción.

De este modo, la ejercitación debe exigirle al sujeto utilizar el sistema de conocimientos declarativos y procedimentales que conforman el contenido de la base orientadora, las microacciones que conforman la acción.

Otro aspecto medular en la elaboración de la base orientadora de la acción es su grado de despliegue. Talízina (1985) plantea que toda nueva acción primero tiene que realizarse en una forma desplegada, para que se hagan conscientes todos sus eslabones $y$, posteriormente, se va abreviando. Del mismo modo, en la asimilación de la base orientadora el grado de independencia que alcanza el sujeto, estará determinado por el tránsito de la acción compartida hacia la acción independiente. 
Figura 1. Aspectos metodológicos para la asimilación de la base orientadora de la acción

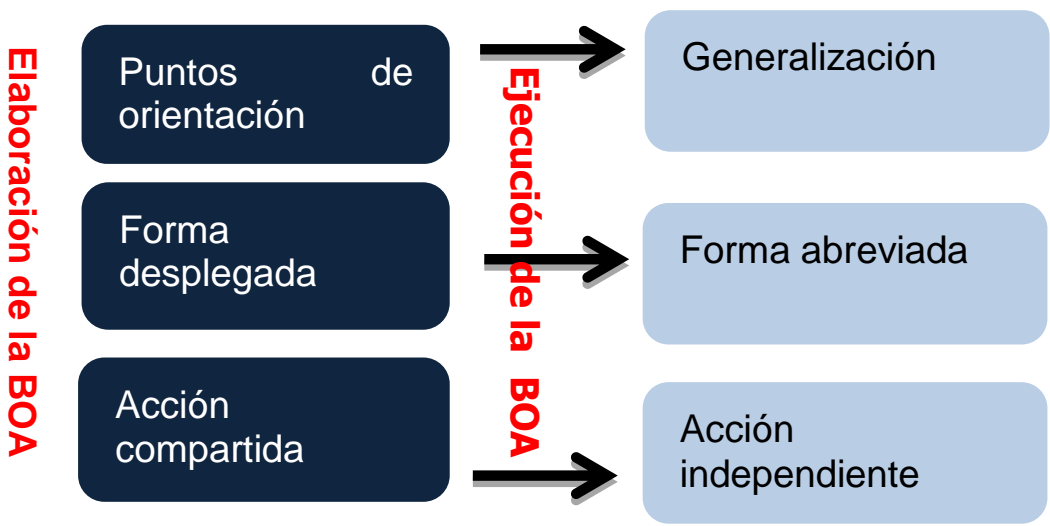

Fuente: elaboración propia, 2014

\subsection{La toma estratégica de apuntes}

La toma de apuntes es el procedimiento más utilizado por los estudiantes universitarios para registrar información relevante en el proceso de aprendizaje, en el cual se emplean de forma simultánea y articulada, habilidades como escuchar, escribir y leer. Monereo y Castelló (1999) señalan la existencia de dos perfiles de anotadores: los estudiantes copistas y los estudiantes estratégicos, tomando en cuenta el uso que le dan a la toma de notas como instrumento y procedimiento de aprendizaje.

Los estudiantes copistas, según Espino y Miras (2010), asumen la toma de notas desde un punto de vista mecanicista, donde la anotación es un medio para reproducir la clase como memoria externa, literal y exhaustiva, requiriendo como condición el ajuste a la velocidad docente. Por su parte, los anotadores estratégicos utilizan las notas como guía del aprendizaje y estas son personalizadas y selectivas, ajustándose a la metodología docente, a la asignatura, al tipo de contenido que se imparte en ella y al tipo de evaluación.

Monereo (2009) señala que la toma de notas de forma estratégica representa un lenguaje construido entre lo externo y lo interno; entre el discurso docente o el contenido de un libro y el discurso propio, al que se acomoda, lo que supone la facilitación de la apropiación e interiorización del contenido, al transcurrir el aprendizaje a través del uso de lenguaje de abreviaturas, símbolos que le son afines al sujeto.

El apunte representa aspectos relevantes de la cultura del aula en la cual se elabora, las palabras apuntadas y la forma en que se anotan expresan determinados acuerdos en 
cuanto a normas, conceptos, procedimientos y valores que resultan significativos en un contexto determinado, el cual es único.

Ahora bien, se puede hablar de una toma estratégica de apuntes, siguiendo a Monereo (2009, p. 84), cuando "un estudiante selecciona la información que debe recoger de forma consciente e intencional, es decir, toma sus decisiones de acuerdo con los objetivos de la tarea que deberá realizar, ajustándose a algunas de las condiciones relevantes del contexto de anotación". De este modo, define 4 dimensiones de la toma estratégica de apuntes en estudiantes universitarios:

- Organización de la información al anotar, tomando en cuenta si el alumnado sigue la misma estructura dada por el profesorado en clase, si realizan una agrupación de contenidos, o si siguen una estructura propia y personalizada, según las características del sujeto.

- Forma en la que se ampliaban los contenidos, si ajustado a las lecturas recomendadas en clase, tomando en cuenta otras asignaturas, o mediante otras fuentes informativas adoptadas por el propio sujeto en su gestión del aprendizaje.

- Reflexión sobre los apuntes, sea de una forma meramente descriptiva, a través de dudas, opiniones y preguntas, o si se produce un posicionamiento argumentado.

- Valoración de la calidad de lo apuntado, tomando en cuenta si la toma de notas se produjo de una manera literal, plasmando todo lo expuesto por el profesorado, si son apuntes basados en una selección de la información, o si hay una personalización en la forma de seleccionar y plasmar los apuntes.

De este modo, siempre que se produzca una toma de notas basada en la personalización de la estructura y de la información misma, adoptando posiciones donde se integre varias fuentes informativas, los apuntes toman una mayor connotación estratégica, y ocurre todo lo contrario cuando los apuntes se enmarcan.

\subsection{Sistema de orientación para la toma estratégica de apuntes}

Un sistema se caracteriza por la interconexión orgánica entre varios componentes, cuyo resultado final es el surgimiento de una cualidad nueva, que no poseen sus partes por separado, y que solo es fruto de esta unión. La realización, además, de cualquier modificación en alguna de sus partes trae como consecuencia la alteración de los restantes, 
y de todo el sistema en general. El sistema que se propone tiene como cualidad nueva la orientación para la toma estratégica de apuntes en estudiantes universitarios.

Asimismo, el sistema de orientación para la toma estratégica de apuntes, se fundamenta en la teoría planificada de las acciones mentales propuesta por Galperin (1982; 1983) y Talízina (1985; 1988), integrando en ella la concepción de aprendizaje estratégico desarrollada por Monereo (Monereo et al. 1996; 1998; 1999).

El sistema de orientación identifica como actividad al proceso encaminado a la solución de tareas por parte del individuo, la cual está integrada por acciones, que a su vez están compuestas por acciones específicas que le dan a la acción forma de proceso continuo, y de cuya sucesión eslabonada depende su realización.

El sistema para la toma estratégica de apuntes, representado en la Figura 2, se estructura en 3 bases orientadoras de la acción (BOA):

- BOA 1: uso estratégico del conocimiento para aprender (Anexo 1)

- BOA 2: técnicas de estudio para tomar apuntes (Anexo 2)

- BOA 3: estrategias para la toma de apuntes (Anexo 3)

El sistema contiene, además, indicaciones metodológicas para la orientación de la toma estratégica de apuntes mediante las direcciones lógicas, pedagógicas y psicológicas.

Para la construcción de las bases orientadoras se partió de la identificación de las acciones necesarias y con un carácter generalizable, así como de los procedimientos concretos que permiten las ejecuciones de las acciones. Se tomó en cuenta las consideraciones de Talízina (1988) acerca de la importancia de identificar las invariantes de las acciones, en la búsqueda de la esencia en la orientación de estas. Así se integraron las invariantes de cada una de las acciones en los contenidos de las bases orientadoras, de manera que en su integración tributen a la toma estratégica de apuntes. Los contenidos de las bases orientadoras fueron construidos a partir de la revisión bibliográfica realizada, para ser presentadas finalmente en el sistema de orientación a través del conocimiento declarativo y procedimental. 
Figura 2. Sistema de orientación para la toma estratégica de apuntes

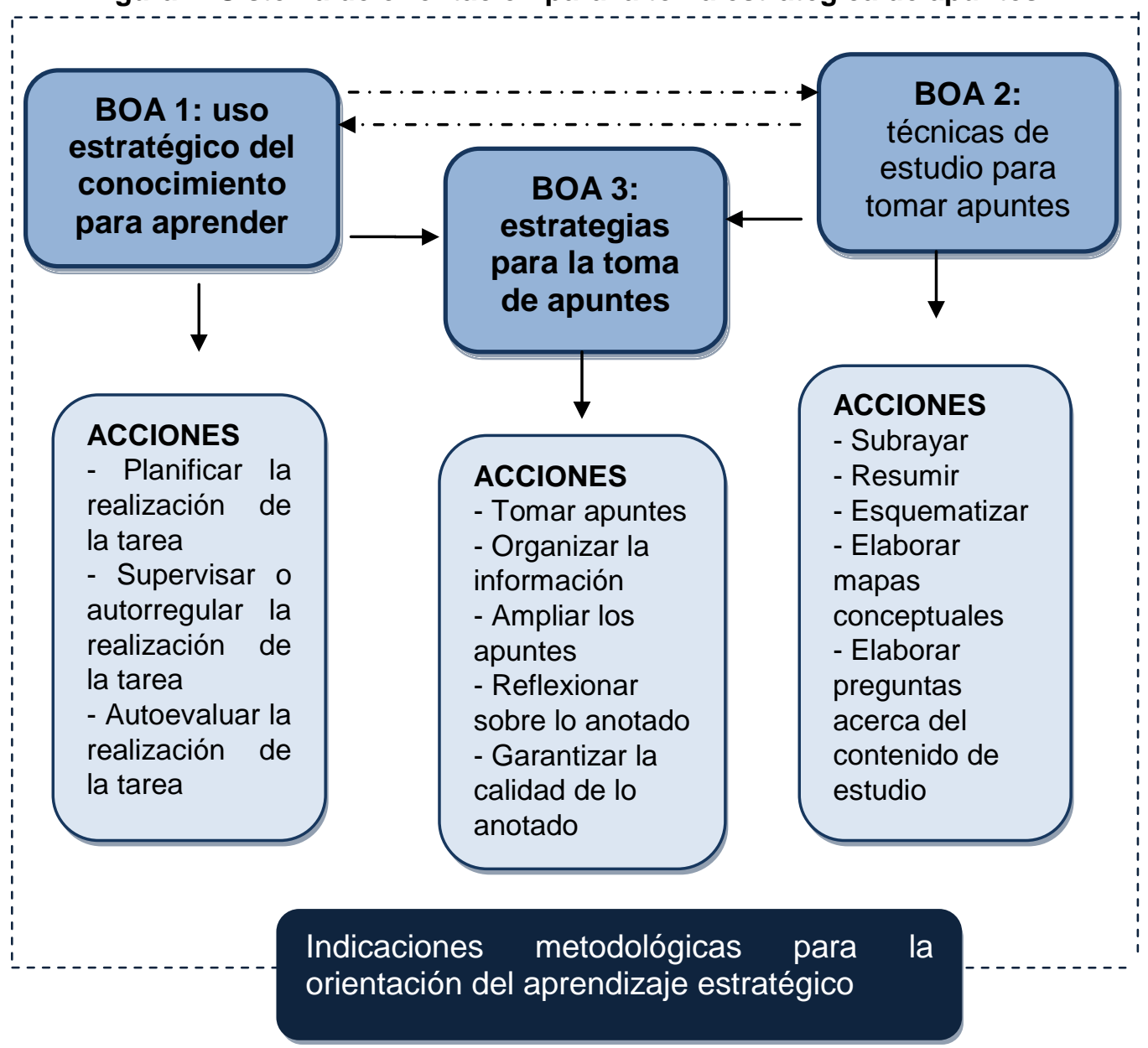

Fuente: elaboración propia, 2013

La base orientadora de la acción uso estratégico del conocimiento para aprender, tiene como intención formar el conocimiento sobre el aprendizaje estratégico. Se tomaron en cuenta para su elaboración, las consideraciones de autores como Monereo, 2009; Monereo et al. (1996); Monereo y Castelló (1999), Monereo y Pérez (1996), Beltrán (2003), Cabrera (2009; 2010), y Pozo, Monereo, Castelló (2001), en relación con las estrategias de aprendizaje y su aplicación, a partir de lo cual se establecieron las acciones y procedimientos necesarios de seguir para su cumplimiento.

En relación con la base orientadora de la acción técnicas de estudio para tomar apuntes, se consideró aportes de autores como Sebastián, Ballesteros y Sánchez (s. f.), a partir de lo cual se identificó un conjunto de técnicas útiles para la realización de la toma de 
apuntes. De estas se elaboró una descripción, una serie de procedimientos y conocimientos requeridos para su efectivo uso.

Finalmente, en la base orientadora de la acción estrategias para la toma de apuntes, se enfatiza la aplicación del aprendizaje estratégico en la toma de apuntes. Para su construcción se hizo uso de la información obtenida en la revisión bibliográfica realizada en función de la toma estratégica de apuntes en estudiantes universitarios. Se tomó en cuenta las posiciones de autores como: Monereo (2009), Monereo y Castelló (1999), y Monereo y Pérez (1996) al respecto, y especialmente, sus consideraciones acerca de las dimensiones sobre la toma estratégica de apuntes.

El sistema de orientación para la toma estratégica de apuntes tiene una organización interna a través de la forma particular de interrelación e interconexión de los elementos que lo componen, lo cual asegura su funcionamiento. Las bases orientadoras para la acción, como componentes establecen relaciones de coordinación al complementarse en el proceso de toma estratégica de apuntes. Del mismo modo, el componente base orientadora de la acción estrategias para la toma de apuntes, establece relaciones de subordinación con los componentes bases orientadoras de la acción, uso estratégico del conocimiento para aprender y técnicas de estudio para tomar apuntes, respectivamente, a las cuales integra en torno a la nueva cualidad.

\section{Indicaciones metodológicas para la orientación de la toma estratégica de apuntes}

\section{Criterios lógicos para la presentación de las bases orientadoras para la acción}

La presentación de las bases orientadoras para la acción debe seguir la lógica de la contribución de cada base al aprendizaje estratégico.

En primer lugar, el estudiante debe recibir las orientaciones sobre el uso estratégico del conocimiento para aprender, base para un aprendizaje estratégico y que se aplicará por su carácter generalizador a cualquier objeto de aprendizaje.

En segundo lugar, el estudiante debe recibir las orientaciones sobre técnicas de estudio que puede emplear para tomar apuntes, según las diferentes situaciones y exigencias de aprendizaje. Las orientaciones sobre las técnicas de estudio deben vincularse a las acciones recibidas por el estudiante sobre el uso estratégico del conocimiento para aprender, de modo que la aplicación de estas técnicas se dimensione estratégicamente en la actividad de aprendizaje. 
En tercer lugar, el estudiante debe recibir las orientaciones sobre las acciones estratégicas para tomar apuntes, las cuales deben vincularse a las orientaciones anteriores sobre el uso estratégico del conocimiento para aprender y las técnicas de estudio.

De este modo, el estudiante se apropia de orientaciones estratégicas en forma de conocimientos declarativo y procedimental, que le permiten en el proceso de toma de apuntes, realizar acciones estratégicas en este sentido, seleccionando y aplicando las técnicas pertinentes y con un proceder estratégico en todo el proceso de toma de apuntes.

\section{Criterios didácticos para la presentación de las bases orientadoras para la acción}

La presentación de las bases orientadoras para la acción debe seguir como criterio didáctico la presentación infusionada del contenido, vinculado a la solución de tareas concretas y garantizando que el estudiante ponga en práctica las acciones, y que realice la actividad de tomar apuntes estratégicamente.

Del mismo modo, se sugiere que la presentación formal de las bases orientadoras se realice a través de tarjetas de estudio para materializar el sistema de orientación como objeto de asimilación a través de sus elementos componentes, sus relaciones y vínculos, y el procedimiento para operar, garantizando en este plano la formación de las acciones mentales. Las tarjetas de estudio pueden ser elaboradas previamente por el profesorado o construidas en conjunto en la etapa de formación del esquema de la base orientadora.

Las tarjetas de estudio deben ser mixtas por su contenido, incluyendo el conocimiento conceptual y los algoritmos procedimentales. Desde el punto de vista formal, deben ser de 3 columnas, incluyendo, respectivamente, objeto de asimilación, composición y control de la ejecución.

Debe combinarse, además, distintos lenguajes como. texto escrito, esquemas, transitando desde un carácter desplegado hasta tarjetas con carácter reducido sobre las acciones.

\section{Criterios psicológicos para la presentación de las bases orientadoras para la acción}

La asimilación como proceso de adquisición de conocimientos y habilidades, demanda la determinadas acciones, partiendo de la presentación de su lógica. El estudiante debe recibir un conocimiento sobre las acciones en sí y sobre las condiciones en las cuales debe 
realizarse la acción. La presentación de las bases orientadoras para la asimilación deberá transitar desde acciones compartidas hasta acciones independientes.

Con respecto al grado de despliegue de la acción, el estudiante tiene que realizar toda nueva acción, primero en una forma desplegada para hacer conscientes todos los eslabones que la integran, transitando hacia su abreviación.

La asimilación de las bases orientadoras debe, además, considerar las etapas del proceso de asimilación y las acciones que realizará el profesorado en cada una de ellas, dirigiendo el aprendizaje de la toma estratégica de apuntes:

1. Formación de la base orientadora: el estudiante debe entrar en contacto con el contenido de la acción, descubriéndose ante él, el material por asimilar mediante la explicación de los aspectos esenciales necesarios para realizar la acción. Las invariantes de las acciones que deben realizarse, se resumen en:

- Mostrar o construir el contenido de la acción.

- Presentar los conocimientos de forma sintetizada.

- Representar el conocimiento en forma externa gráfica, en forma de esquema que incluya los aspectos lógicos necesarios para realizar la acción.

- Representar la lógica de la acción en su totalidad.

- Proyectar las acciones hacia aquellas tareas problemas que es necesario resolver.

- Representación por los sujetos del esquema de la base orientadora de la acción. Debe responderse a la pregunta: ¿Existe claridad sobre la actuación, los conocimientos necesarios (rasgos esenciales) para ejecutar la acción? La comprensión del esquema no significa que se haya asimilado la base orientadora.

2. Acciones externas materializadas: os sujetos aprenden las acciones externas materializadas a través de la solución de problemas o tareas concretas. Lo esencial es que el conocimiento se represente de forma externa mediante diferentes recursos (pizarra, diapositivas, dispositivos cognitivos, tarjetas de estudio), donde se plasma el esquema de la base orientadora de la acción, el cual se utiliza en la solución de la tarea. Ahora bien, la forma gráfica externa debe representar solamente lo que refleja la esencia del contenido o de la acción. Las tareas deben reflejar casos típicos de aplicación de la acción dada, y el número de tareas no debe responder a un solo tipo para evitar la reducción y automatización.

3. Trabajo independiente: el profesorado deviene en mecanismo de regulación que dirige la acción del alumnad, controlando los pasos de la acción. El objetivo central es 
asimilar el contenido operacional, utilizándose tareas que respondan a diferentes situaciones donde deba ser aplicado el conocimiento.

Acción en forma de lenguaje sin apoyo de acciones externas: predominio de las acciones en forma de lenguaje, sin apoyo de acciones externas materializadas, de modo que los sujetos que aprenden expresen todo de forma oral. Resulta esencial el trabajo en parejas, donde se intercambian roles que consisten en la explicación de las soluciones en voz alta y el control de lo expresado, registrándose los errores. De este modo, el sujeto transita del control externo al interno, aprende a controlarse en la medida que controla a otro.

4. Acción mental: comprende la interiorización de formas abreviadas de la acción mental, posibilitando el tránsito de los eslabones de la acción hacia el lenguaje interno, por lo que deviene en un acto del pensamiento.

\section{Conclusiones}

La orientación del aprendizaje para la toma estratégica de apuntes en estudiantes universitarios, se comprende a través de la teoría planificada de las acciones mentales. Esta se sustenta en 4 condiciones básicas: la formación de una adecuada motivación de las acciones, el aseguramiento de la correcta ejecución de la nueva acción, la formación de las propiedades deseadas de la nueva acción, y la traslación de la acción al plano mental, lo cual se logra mediante el paso de la acción por una serie de etapas: la material externa, la verbal externa, y la verbal interna. Dentro de la dirección de la acción, ocupa un papel muy importante la orientación o base orientadora, la cual constituye el sistema de condiciones en el cual se apoya el individuo para cumplir la acción.

Un sistema de orientación del aprendizaje para la toma estratégica de apuntes en estudiantes universitarios, debe integrar como bases orientadoras, el uso estratégico del conocimiento para aprender, las técnicas de estudio para tomar apuntes, las estrategias para la toma de apuntes, así como recomendaciones metodológicas que pauten su presentación desde requerimientos lógicos, didácticos y psicológicos. La base orientadora estrategias para la toma de apuntes, constituye el eje que integra las otras bases orientadoras en el sistema de orientación, en torno a la toma estratégica de apuntes. 


\section{Referencias}

Beltrán, Jesús A. (2003). Estrategias de aprendizaje. Revista de Educación, (332), 55-73.

Cabrera Ruiz, Isaac. (2009). Autonomía en el aprendizaje: Direcciones para el desarrollo en la formación profesional. Actualidades Investigativas en Educación, 9(2), 1-22. Recuperado de http://revista.inie.ucr.ac.cr/uploads/tx magazine/aprendizaje.pdf

Castellanos Simons, Doris, Castellanos Simons, Beatriz, Llivina Lavigne, Miguel Jorge y Silverio Gómez, Mercedes. (2001). Hacia una concepción del aprendizaje desarrollador. La Habana: Instituto Superior Pedagógico "Enrique José Varona".

Coll, Cesar. (1996). Aprendizaje escolar y construcción del conocimiento. Barcelona: Paidos.

Domínguez García, Laura. (2005). Psicología del desarrollo. Problemas, principios y categorías. La Habana: Editorial Félix Varela.

Eguino de la Paz, Yoisel. (2013). Sistema de orientación para la toma estratégica de apuntes en estudiantes universitarios. (Trabajo de diploma no publicado). Universidad Central "Marta Abreu" de Las Villas, Villa Clara, Cuba.

Espino, Sandra y Miras, Mariana. (2010). El uso de los apuntes como instrumentos de aprendizaje: un análisis de casos. Lectura y vida, 31(4), 27-34.

Galperin, Pior. (1982). Introducción a la psicología. La Habana: Editorial Pueblo y Educación.

Galperin, Pior. (1983). Sobre la formación de los conceptos y las acciones mentales. En Ana Luisa Segarte (ed.), Lecturas de psicología pedagógica (pp. 139-156). Facultad de Psicología: Universidad de La Habana.

Galperin, Pior (2003). Sobre la investigación del desarrollo intelectual del niño. En Psicología del desarrollo. Selección de lecturas (pp. 247-263). La Habana: Félix Varela.

González Clavero, María Victoria. (2012). Autonomía para aprender: asignatura para el currículo optativo/electivo de la Educación Superior. (Tesis de maestría no publicada). Universidad Central "Marta Abreu" de Las Villas, Villa Clara, Villa Clara, Cuba.

González Rey, Fernando. (1986). Las operaciones cognitivas de la personalidad. Estado actual de su investigación en la psicología marxista. Revista Cubana de Psicología, 3(3), 16-25.

González Rey, Fernando. (1997). Epistemología cualitativa y subjetividad. La Habana: Editorial Pueblo y Educación.

Martínez, Graciela. (2003). Algunos aspectos fundamentales de la teoría de la formación planificada por etapas de las acciones mentales de P. Ya. Galperin. Psicología del desarrollo. Selección de lecturas (pp. 237-242). La Habana Félix Varela.

Marugán, Monserrat, Martín, Luis Jorge, Catalina, Javier y Román, José María. (2013). Estrategias cognitivas de elaboración y naturaleza de los contenidos en estudiantes universitarios. Psicología Educativa, 19(1), 13-20. 
Menchinskaya, Natalia, y Sabutorova, Galina. (1988). Problemas teóricos del desarrollo psíquico del niño. En Selección de lecturas de psicología de las edades I (Vol. 3, pp.47114). La Habana: Empresa Nacional de Producción del Ministerio de educación Superior.

Monereo, Carles. (1990). Las estrategias de aprendizaje en la Educación Formal: Enseñar a pensar y sobre el pensar. Infancia y Aprendizaje, (50), 3-25.

Monereo, Carles. (1997). La construcción del conocimiento estratégico en el aula. En María Luisa Pérez (coord.), La enseñanza y el aprendizaje de estrategias desde el currículo (pp. 21-34). Girona, España: Editorial Horsori.

Monereo, Carles. (2009). Aprender a encontrar y seleccionar información: de "Google" a la toma de apuntes. En Juan Ignacio Pozo y María del Puy Pérez Echevarría (coords.), Psicología del aprendizaje universitario. La formación en competencias (pp. 89-105). Madrid: Morata.

Monereo, Carles y Castelló, Monserrat. (1999). Esto hay que apuntarlo. La toma de notas como estrategia de aprendizaje. Recuperado el 12 de abril de 2013, de http://redaberta.usc.es/aidu/index2.php?option=com docman\&task=doc view\&gid=107 $\underline{\text { \&ltemid }=8}$.

Monereo, Carles, Castelló, Monserrat, Clariana, Mercé, Palma, Monserrat, y Pérez, María. (1997). Estrategias de enseñanza y aprendizaje. Formación del profesorado y aplicación en el aula (3a. ed.). España: Editorial Graó.

Monereo, Carles y Pérez, María. (1996) La incidencia de la toma de apuntes sobre el aprendizaje significativo. Un estudio en enseñanza superior. Infancia y Aprendizaje, 73, 65-86.

Morin, Edgar (1999). Los siete saberes necesarios a la educación del futuro. París: UNESCO.

Nieves, Zaida, Otero, Idania, y Molerio, Osana. (2007). La formación profesional en la universidad de hoy: de la educación a la autoeducación. Revista Pedagogía Universitaria 12(2), 1-9.

Palma, Monserrat. (1997). La motivación del estudiante y la construcción del conocimiento estratégico. En María Luisa Pérez Cabani (coord.), La enseñanza y el aprendizaje de estrategias desde el curriculum (Cuadernos para el Análisis 10, pp. 35-47). Universidad de Girona. Barcelona: HORSORI.

Poggioli, Lisette. (1989). Estrategias cognoscitivas: una revisión teórica y empírica. En Aníbal Puente, Lisette Poggioli y Armando Navarro (coords.). Psicología cognoscitiva (pp.277322). Venezuela: Editorial McGraw- Hill.

Pozo, Juan Ignacio y Monereo, Carles. (1999). El aprendizaje estratégico. Enseñar a aprender desde el currículo. Madrid: Santillana/Aula XXI. 
Pozo, Juan Ignacio, Monereo, Carles y Castelló Monserrat. (2001). El uso estratégico del conocimiento. En César Coll, Jesús Palacios y Álvaro Marchesi (comp.), Psicología de la educación escolar (pp. 211-234). Madrid: Alianza Editorial.

Santana, Lidia. (2009). Aprender a aprender: el viejo desideratum del sistema educativo. Recuperado http://www.quadernsdigitals.net/index.php?accionMenu=hemeroteca.VisualizaArticulol $\underline{\text { U.visualiza\&articulo } \mathrm{id}=1268}$

Sebastián, Araceli, Ballesteros, Belén y Sánchez Mari Fe. (s.f.). Técnicas de estudio. España: Universidad Nacional de Educación a Distancia. Recuperado de http://portal.uned.es/pls/portal/docs/PAGE/UNED MAIN/OFERTA/NUEVOS\%20ESTU DIANTES/PLAN ACOGIDA ESTUDIANTES/GUIAS\%20PARA\%20EL\%20ENTRENA MIENTO\%20DE\%20COMPETEN/PLAN ACOGIDA GUIAS APOYO TECNICAS ES TUDIO COIE.PDF

Talízina, Nidia. (1985). Conferencias sobre los fundamentos de la enseñanza en la educación superior. La Habana: Departamento de Estudios para el Perfeccionamiento de la Educación Superior.

Talízina, Nidia. (1988). Psicología de la enseñanza. Moscú: Editorial Progreso.

Tünnermann, Carlos. (2010). La educación permanente y su impacto en la educación superior. Revista Iberoamericana de Educación Superior (RIES), 1(1), 120-133. 


\section{Anexo 1 Base orientadora de la acción: uso estratégico del conocimiento para aprender}

\section{Conocimiento declarativo}

El uso estratégico del conocimiento exige planificar la realización de la tarea, supervisar su realización y la autoevaluación de la ejecución.

Planificar la realización de la tarea implica la comprensión de las exigencias que contiene su cumplimiento, la identificación de factores como el tiempo con que se dispone, la disposición de los recursos necesarios, los principios a los cuales se ajustará la evaluación de los resultados, así como la evaluación de los conocimientos que posee la persona, útiles para la resolución de la tarea, aquellos que aun necesita conocer y el modo de encontrarlos.

La supervisión de la realización de la tarea consiste en el control del ajuste constante a las condiciones de la tarea, a partir de la valoración con respecto a la aproximación al objetivo perseguido, control del tiempo y el empleo de los recursos necesarios.

La autoevaluación de la realización de la tarea consiste en la valoración efectuada por el estudiante del cumplimiento de los objetivos planteados, la evaluación del proceso de ajuste a las condiciones, la identificación de dificultades, así como la forma de realizarla.

\section{Conocimiento procedimental}

1.Planificar la realización de la tarea

$>$ Identificar las condiciones relevantes de la tarea que se debe aprender o resolver.

$>$ Identificar el resultado que se debe obtener.

$>$ Conocer la forma en la que se evaluará dicho resultado.

$>$ Identificar conocimientos previos que puedan ayudar a enfrentar la tarea.

$>$ Analizar las técnicas y procedimientos que puedan ayudar a lograr los objetivos.

2. Supervisar o autorregular la realización de la tarea

$>$ Ajustarse a las condiciones de la tarea.

$>$ Valorar la pertinencia de las técnicas y procedimientos que se aplican.

$>$ Evaluar el progresivo acercamiento a los objetivos de forma correcta.

$>$ Seleccionar alternativas ante las dificultades que aparezcan en el proceso.

$>$ Identificar nuevos procedimientos que se puedan aplicar para la realización de la tarea.

3. Autoevaluar la tarea realizada

$>$ Valorar el ajuste a las condiciones que exigía la tarea durante su ejecución.

$>$ Valorar el alcance de los resultados esperados.

$>$ Evaluar la superación satisfactoria de las dificultades encontradas durante el proceso.

$>$ Identificar nuevas formas de interpretar las demandas y criterios de evaluación, al efectuar una tarea similar.

$>$ Valorar la pertinencia de las técnicas o procedimientos empleados.

$>$ Identificar nuevos procedimientos que utilizaría en una nueva oportunidad. 


\section{Anexo 2 Base orientadora de la acción para el empleo de técnicas de estudio con el fin de tomar apuntes}

La toma de apuntes exige el empleo de técnicas que permitan captar la información y convertirla en anotaciones, según las características de la información, las condiciones del proceso y las particularidades de quien anota. Entre las técnicas más efectivas que pueden emplearse se identifican: el resumen, la esquematización, la elaboración de preguntas acerca del contenido de estudio, la elaboración de mapas conceptuales y el subrayado.

\section{Técnica de estudio Subrayar}

\section{Conocimiento declarativo}

El subrayado es una importante técnica de selección de información, caracterizada por la sencillez en el procedimiento y su gran efectividad. Es una de las técnicas más utilizadas en el aula, ya que contribuye a la organización y a la asimilación del contenido anotado.

\section{Conocimiento procedimental}

$>$ Leer el contenido anotado totalmente.

$>$ Seleccionar las ideas más importantes.

$>$ Subrayar palabras y frases claves dentro de esas ideas importantes.

$>$ Evitar subrayar conjunciones, preposiciones o artículos.

$>$ Utilizar códigos personales para resaltar la información.

\section{Técnica de estudio Resumir}

\section{Conocimiento declarativo}

Es una forma de condensar información, de sintetizar ideas del contenido, de forma tal que se expresa lo manifiesto en este último, con las propias palabras de quien lo elaboró, a manera de extracto de la información original. Este se construye a través de la extracción de las ideas fundamentales.

\section{Conocimiento procedimental}

$>$ Analizar el documento o comunicación.

Encontrar la idea central de cada párrafo.

$>$ Darle a cada idea una prioridad de acuerdo con su importancia.

Detectar las relaciones entre estas ideas.

\section{Técnica de estudio Esquematizar}

\section{Conocimiento declarativo}

Un esquema se construye a través de la selección de las ideas indispensables de determinado contenido. Adopta una forma breve y sintética al expresar las ideas fundamentales de determinada información, de una forma fluida y precisa, pudiéndose construir mediante diferentes estructuras.

\section{Conocimiento procedimental}

$>$ Identificar ideas fundamentales y sus relaciones.

$>$ Representar dichas ideas a través de una estructura visual: esquemas de llaves, esquemas numéricos, esquemas de subordinación, cuadro sinóptico. 


\section{Técnica de estudio Elaborar mapas conceptuales}

\section{Conocimiento declarativo}

Los mapas conceptuales constituyen una técnica gráfica que establece representaciones jerárquicas entre los conceptos relativos a un área de conocimiento. Permite valorar los conocimientos previos y relacionarlos con varios marcos de conocimiento.

\section{Conocimiento procedimental}

$>$ Estudiar con atención el contenido.

$>$ Seleccionar los conceptos más importantes.

- Elaborar una lista de conceptos, ordenándolos de los más generales a los particulares.

$>$ Realizar la jerarquía conceptual.

$>$ Formar proposiciones.

$>$ Construir el mapa.

$>$ Leer el mapa como si fuera una narración.

\section{Técnica de estudio Elaborar preguntas acerca del contenido de estudio}

Conocimiento declarativo

La elaboración de preguntas sobre determinado contenido es un ejercicio de reflexión de lo estudiado, que permite, además, la interiorización del contenido. Constituye una ayuda al estudiante, ya que favorece la comprensión de la información y fortalece así sus modos de expresión.

Conocimiento procedimental

$>$ Identificar objeto y contenido de estudio y su importancia.

$>$ Dirigir la atención a la búsqueda de información sobre el objeto y contenido de estudio, a partir de la comprensión acerca de lo que se tiene y lo que falta por conocer.

> Elaborar preguntas que demuestren un conocimiento acerca del contenido tratado, o una comprensión de este, posibilitando la profundización a partir de la problematización.

- Elaborar preguntas sobre lo que no domina.

Analizar las preguntas elaboradas y las posibles respuestas.

- Evaluar lo realizado. 


\section{Anexo 3 Base orientadora de la acción: estrategias para la toma de apuntes}

\section{Conocimiento declarativo}

La toma apuntes desde una posición estratégica, exige que las notas sean organizadas, se amplíen con nueva información, sean objeto de reflexión y se garantice su calidad.

Para la toma de apuntes se puede seguir varios formatos, ya sea a través de bloques o columnas, así como gráficas o esquemas, poniendo un mayor énfasis en el impacto visual. En esta forma de tomar datos se brinda la posibilidad de almacenar y codificar la información importante de determinado contenido, a través de la selección de sus ideas fundamentales, pudiendo además agregar comentarios valorativos personales, y teniendo la oportunidad luego de complementar dicha información con nuevos datos.

La organización de la información anotada es un elemento básico que garantiza la utilidad de los apuntes, al facilitar la codificación de la información recibida y por constituir una fuente para posteriores consultas. No existen esquemas para realizar la organización de la información de una forma correcta, siendo la estructura personalizada la más recomendada.

La ampliación o complementación de la información recibida constituye un elemento esencial, ya que contribuye a la reflexión de la persona que la realiza. En este sentido, es importante no solo añadir datos relacionados con asignaturas afines a las que dio origen a la nota, si no consultar diversidad de fuentes para lograr la integración con otros contenidos y estimular procesos cognitivos, como el pensamiento.

Reflexionar sobre el contenido anotado contribuye al aprendizaje lógico de la información, y a la aprehensión de las ideas fundamentales a través de la comprensión y el razonamiento.

La calidad de los apuntes es una premisa indispensable para aprender estratégicamente. Realizar apuntes selectos y sintéticos, revisarlos y contrastarlos con otros, garantiza que la información obtenida sea no solo la correcta, si no la esencial, facilitando de esta forma su asimilación.

\section{Conocimiento procedimental}

\section{Tomar apuntes}

$>$ Identificar la fuente de información a partir de la cual se tomará los apuntes (fuente oral o escrita; profesor en clases, libros, informes, por ejemplo).

$>$ Concentrar la atención en dicha fuente de apuntes.

$>$ Realizar una lectura minuciosa o escucha atenta del material del cual se va a anotar.

$>$ Buscar el significado de lo que se dice o lee.

$>$ Identificar el tema tratado y qué se dice del mismo.

$>$ Apuntar de forma breve las ideas claves.

$>$ Anotar textualmente las definiciones.

$>$ Dejar espacios para aclaraciones.

> Hacer uso de abreviaturas y símbolos propios del sujeto.

> Subrayar las ideas o palabras claves.

$>$ Comparar notas con las de los otros estudiantes, analizando si falta algún contenido fundamental.

> Apoyarse en resúmenes, esquemas, mapas conceptuales para tratar temas complejos.

$>$ Dejar espacios en blanco para enriquecer las notas. 


\section{Organizar la información}

Analizar la información obtenida.

- Agrupar los contenidos en correspondencia con la relación que se establece entre ellos.

> Seleccionar técnicas de recogida y organización de la información (tablas; esquemas; cuadros sinópticos, resúmenes, entre otros).

\section{Ampliar los apuntes}

Releer la información tomada.

Valorar la información tomada en cuanto a su plenitud.

- Identificar información que pudiera complementar la información que ya se tiene.

> Consultar diversas fuentes de información para ampliar los apuntes (bibliografía de la asignatura, de materias afines, entre otros).

Relacionar la información de los apuntes con otros contenidos.

\section{Reflexionar sobre lo anotado}

Analizar la información tomada.

- Plantear dudas, preguntas acerca de esta información.

> Tomar una posición con respecto a los apuntes y argumentarla.

\section{Operación: garantizar la calidad de lo anotado}

> Seleccionar la información al anotar, según su importancia.

$>$ Sintetizar el contenido por anotar.

$>$ Revisar los apuntes una vez concluida su realización. 